Journal of Mathematics and Statistics 6 (3): 306-315, 2010

ISSN 1549-3644

(C) 2010 Science Publications

\title{
Cost Analysis of a Two Dissimilar-Unit Cold Standby Redundant System Subject to Inspection and Random Change in Units
}

\author{
${ }^{1}$ G.S. Mokaddis and ${ }^{2}$ C.H. Matta \\ ${ }^{1}$ Department of Mathematics, Faculty of Science, \\ Ain Shams University, Cairo, Egypt \\ ${ }^{2}$ Department of Statistics, Faculty of Economics and Political Sciences, \\ Cairo University, Cairo, Egypt
}

\begin{abstract}
Problem statement: This study deled with the cost analysis of a two dissimilar unit cold standby redundant system subject to inspection and random change in units. In this system each unit works in two different modes normal and total failure. Assuming that the failure, repair, post repair, interchange of units and inspection times are stochastically independent random variables each having an arbitrary distribution. Approach: The system was analyzed by semi Markov process technique. Results: The time-dependent availability, steady-state availability, busy period analysis, expected number of visits by the repairman were obtained numerically and cost analysis was obtained numerically and graphically. Conclusion: Expected cost per unit time decreased with respect to the increase of failure rate.
\end{abstract}

Key words: Availability, busy period, expected number of visits by the repairman, cost analysis

\section{INTRODUCTION}

Several authors have analyzed two-unit redundant system with two states of operation-operative and failed. Models have been formulated to treat many situations. The system analyses by the semi-Markov process technique. The transition probabilities, mean sojourn time and the mean time to system failure has been obtained by (Mokaddis et al., 1997; 2010).

The purpose of the present study is to study the pointwise availability, steady state availability, busy period, expected number of visits and cost per unit time of the system. This study deals with a model of a two dissimilar-unit cold standby redundant system with two modes normal and failure, were a single repair facility is available with the system for inspection, repair and post repair of the failed unit. The operative and standby units are interchanged at random times to achieve high reliability of the system. After repair, the unit is sent for inspection to decide whether the repair is satisfactory or not. If the failed unit is found unsatisfactory on inspection then the unit sent to pose repair. After repair or post repair of the failed unit it becomes like a new one. The results obtained by (Goel et al., 1992) are derived from this study as special cases.

The following assumptions and notations are used to analyze the system:
- The system consists of a two-dissimilar units, the first is operative and the second is kept as cold standby, which of course does not fail unless it goes into operation

- A unit has two possible modes-normal (the unit functions with full capacity) and total failure (the unit capacity is reduced below the specified level)

- After a random amount of time, the operative unit becomes standby and the standby unit becomes operative if the standby is available

- After failure of an operative unit, the cold standby unit becomes operative instantaneously

- $\quad$ Failure, repair, post repair, interchange of units and inspection times are stochastically independent random variables each having an arbitrary distribution

- There is only one repair facility available with the system to repair, inspection and post repair of the failed unit

- After repair, a unit goes for inspection to decide whether the repair is satisfactory or not, if the repaired unit is found to be unsatisfactory then it is sent for post repair

- The probability of having satisfactory repair is fixed

- On repair or post repair a unit acts like a new unit

- Service discipline is first come first served

- All random variables are mutually independent 


\section{Notations and states of the system:}

$\mathrm{E}_{0} \quad$ State of the system at epoch $\mathrm{t}=0$

E set of regenerative states; $\left\{S_{0}, S_{1}, S_{2}, S_{3}\right.$, $\left.\mathrm{S}_{4}, \mathrm{~S}_{5}, \mathrm{~S}_{6}, \mathrm{~S}_{7}\right\}$

$\overline{\mathrm{E}} \quad$ set of non-regenerative states; $\left\{\mathrm{S}_{8}, \mathrm{~S}_{9}, \mathrm{~S}_{10}\right.$, $\left.\mathrm{S}_{11}, \mathrm{~S}_{12}, \mathrm{~S}_{13}\right\}$

$f_{i}(t), F_{i}(t) \quad$ Pdf and cdf of failure time of the $i$-th unit; $\mathrm{i}=1,2$

$\ell_{\mathrm{i}}(\mathrm{t}), \mathrm{L}_{\mathrm{i}}(\mathrm{t}) \quad$ Pdf and cdf of time after thew $\mathrm{i}$-th operative unit changes; $i=1,2$

$\mathrm{g}_{\mathrm{i}}(\mathrm{t}), \mathrm{G}_{\mathrm{i}}(\mathrm{t}) \quad$ Pdf and cdf of repair time of the i-th failed unit; $i=1,2$

$\mathrm{h}_{\mathrm{i}}(\mathrm{t}), \mathrm{H}_{\mathrm{i}}(\mathrm{t}) \quad$ Pdf and cdf of time to complete inspection of the $i$-th failed unit; $i=1,2$

$\mathrm{k}_{\mathrm{i}}(\mathrm{t}), \mathrm{K}_{\mathrm{i}}(\mathrm{t}) \quad$ Pdf and cdf of time to complete post repair of the $\mathrm{i}$-th unsatisfactory failed unit; $i=1,2$

$p_{i}=\left(1-q_{i}\right) \quad$ Probability that the repair of the i-th unit is satisfactory after the inspection; $i=1,2$

$\mathrm{q}_{\mathrm{ij}}(\mathrm{t}), \mathrm{Q}_{\mathrm{ij}}(\mathrm{t}) \quad$ Pdf and cdf of first passage time from regenerative state $i$ to a regenerative state $\mathrm{j}$ or to a failed state $\mathrm{j}$ without visiting any other regenerative state in $(0, \mathrm{t}] ; \mathrm{i}, \mathrm{j} \in \mathrm{E}$

$q_{i j}^{(k)}(t), Q_{i j}^{(k)}(t)$ Pdf and cdf of first passage time from regenerative state $i$ to a regenerative state $\mathrm{j}$ or to a failed state $\mathrm{j}$ visiting state $\mathrm{k}$ only once in $(0, t] ; i, j \in E, k \in \bar{E}$

$\mathrm{p}_{\mathrm{ij}} \quad$ One step transition probability from state $i$ to state $j ; i, j \in E$

$p_{i j}^{(k)} \quad$ Probability that the system in state $i$ goes to state $\mathrm{j}$ passing through state $\mathrm{k} ; \mathrm{i}, \mathrm{j} \in \mathrm{E}$; $\mathrm{k} \in \overline{\mathrm{E}}$

$\pi_{\mathrm{i}}(\mathrm{t}) \quad$ Cdf of first passage time from regenerative state $i$ to a failed state

$\mathrm{M}_{\mathrm{i}}(\mathrm{t}) \quad$ Probability that the system having started from state $i$ is up at time $t$ without making any transition into any other regenerative state

$\mathrm{B}_{\mathrm{i}}(\mathrm{t}) \quad$ Probability that the server is busy at time $\mathrm{t}$ given that the system entered regenerative state $i$ at time $t=0$

$\mathrm{V}_{\mathrm{i}}(\mathrm{t}) \quad$ Expected number of visits by the server given that the system started from regenerative state $\mathrm{i}$ at time $\mathrm{t}=0$

$\mu_{\mathrm{ij}} \quad$ Contribution mean sojourn time in state $\mathrm{i}$ when transition is to state $\mathrm{j}$ is

$-\tilde{\mathrm{Q}}_{\mathrm{ij}}(0)=\mathrm{q}_{\mathrm{ij}}^{*}(0)$

$\mu_{\mathrm{i}} \quad$ Mean sojourn time in state i, $\mu_{\mathrm{i}}=$ $\sum_{\mathrm{j}}\left[\mu_{\mathrm{ij}}+\sum_{\mathrm{k}} \mu_{\mathrm{ij}}^{(\mathrm{k})}\right]$
Symbol for Laplace-Stieltjes transform, e.g., $\tilde{F}(\mathrm{~s})=\int \mathrm{e}^{-\mathrm{st}} \mathrm{dF}(\mathrm{t})$

* $\quad$ Symbol for Laplace transform, e.g., $\mathrm{f}^{*}(\mathrm{~s})=\int \mathrm{e}^{-\mathrm{st}} \mathrm{f}(\mathrm{t}) \mathrm{dt}$

s Symbol for Stieltjes convolution, e.g.,

$\mathrm{A}(\mathrm{t}) \mathrm{s} \mathrm{B}(\mathrm{t})=\int_{0}^{\mathrm{t}} \mathrm{B}(\mathrm{t}-\mathrm{u}) \mathrm{dA}(\mathrm{u})$

(C) Symbol for ordinary convolution, e.g., $a(t) \subseteq b(t)=\int_{0}^{t} a(u) b(t-u) d u$

For simplicity, whenever integration limits are $(0, \infty)$, they are not written.

Symbols used for the states:

$\mathrm{N}_{\text {oi }}$ The $\mathrm{i}$-th unit is operative in normal mode; $\mathrm{i}=1,2$

$\mathrm{N}_{\mathrm{Si}}$ The $\mathrm{i}$-th unit is standby in normal mode; $\mathrm{i}=1,2$

$F_{w r i}$ The $i$-th unit is in total failure mode and waiting for repair; $i=1,2$

$\mathrm{F}_{\mathrm{ri}}$ The $\mathrm{i}$-th unit is in total failure mode and under repair; $i=1,2$

$\mathrm{F}_{\mathrm{Ri}}$ The $\mathrm{i}$-th unit is in total failure mode with repair continued from earlier state; $i=1,2$

$\mathrm{F}_{\mathrm{Ii}}$ The i-th unit is in total failure mode and under inspection; $i=1,2$

$F_{\text {pri }}$ The i-th unit is in total failure mode and under post repair after inspection; $i=1,2$

Considering these symbols, the system may be in one of the following states:

$\mathrm{S}_{0} \equiv\left(\mathrm{N}_{\mathrm{o} 1}, \mathrm{~N}_{\mathrm{S} 2}\right), \quad \mathrm{S}_{1} \equiv\left(\mathrm{N}_{\mathrm{S} 1}, \mathrm{~N}_{\mathrm{o} 2}\right), \quad \mathrm{S}_{2} \equiv\left(\mathrm{F}_{\mathrm{r} 1}, \mathrm{~N}_{\mathrm{o} 2}\right)$,

$\mathrm{S}_{3} \equiv\left(\mathrm{N}_{\mathrm{o} 1}, \mathrm{~F}_{\mathrm{r} 2}\right), \quad \mathrm{S}_{4} \equiv\left(\mathrm{F}_{\mathrm{r} 1}, \mathrm{~N}_{\mathrm{o} 2}\right), \quad \mathrm{S}_{5} \equiv\left(\mathrm{N}_{\mathrm{o} 1}, \mathrm{~F}_{\mathrm{r} 2}\right)$,

$\mathrm{S}_{6} \equiv\left(\mathrm{F}_{\mathrm{pr} 1}, \mathrm{~N}_{\mathrm{o} 2}\right), \quad \mathrm{S}_{7} \equiv\left(\mathrm{N}_{\mathrm{o} 1}, \mathrm{~F}_{\mathrm{pr} 2}\right), \quad \mathrm{S}_{8} \equiv\left(\mathrm{F}_{\mathrm{R} 1}, \mathrm{~F}_{\mathrm{wr} 2}\right)$,

$\mathrm{S}_{9} \equiv\left(\mathrm{F}_{\mathrm{wr} 1}, \mathrm{~F}_{\mathrm{R} 2}\right) \quad \mathrm{S}_{10} \equiv\left(\mathrm{F}_{\mathrm{I} 1}, \mathrm{~F}_{\mathrm{wr} 2}\right), \quad \mathrm{S}_{11} \equiv\left(\mathrm{F}_{\mathrm{wr} 1}, \mathrm{~F}_{\mathrm{I} 2}\right)$,

$\mathrm{S}_{12} \equiv\left(\mathrm{F}_{\mathrm{pr} 1}, \mathrm{~F}_{\mathrm{wr} 2}\right), \quad \mathrm{S}_{13} \equiv\left(\mathrm{F}_{\mathrm{wr}}, \mathrm{F}_{\mathrm{pr} 2}\right)$

Up states: $S_{0}, S_{1}, S_{2}, S_{3}, S_{4}, S_{5}, S_{6}, S_{7}$.

Down states: $S_{8}, S_{9}, S_{10}, S_{11}, S_{12}, S_{13}$.

States and possible transitions between them are shown in Fig. 1.

Availability analysis: From arguments used in the theory of regenerative processes, the point wise availabilities $A_{i}(t)$ are seen to satisfy the following relations: 


$$
\begin{aligned}
& \mathrm{A}_{0}(\mathrm{t})=\mathrm{M}_{0}(\mathrm{t})+\mathrm{q}_{01}(\mathrm{t}) \odot \mathrm{A}_{1}(\mathrm{t})+\mathrm{q}_{02}(\mathrm{t}) \odot \mathrm{A}_{2}(\mathrm{t}), \\
& \mathrm{A}_{1}(\mathrm{t})=\mathrm{M}_{1}(\mathrm{t})+\mathrm{q}_{10}(\mathrm{t}) \odot \mathrm{A}_{0}(\mathrm{t})+\mathrm{q}_{13}(\mathrm{t}) \odot \mathrm{A}_{3}(\mathrm{t}), \\
& \mathrm{A}_{2}(\mathrm{t})=\mathrm{M}_{2}(\mathrm{t})+\mathrm{q}_{24}(\mathrm{t}) \odot \mathrm{A}_{4}(\mathrm{t})+\mathrm{q}_{2,10}^{(8)}(\mathrm{t}) \odot \mathrm{A}_{10}(\mathrm{t}), \\
& \mathrm{A}_{3}(\mathrm{t})=\mathrm{M}_{3}(\mathrm{t})+\mathrm{q}_{35}(\mathrm{t}) \odot \mathrm{A}_{5}(\mathrm{t})+\mathrm{q}_{3,11}^{(9)}(\mathrm{t}) \odot \mathrm{A}_{11}(\mathrm{t}), \\
& \mathrm{A}_{4}(\mathrm{t})=\mathrm{M}_{4}(\mathrm{t})+\mathrm{q}_{41}(\mathrm{t}) \odot \mathrm{A}_{1}(\mathrm{t})+\mathrm{q}_{46}(\mathrm{t}) \odot \mathrm{A}_{6}(\mathrm{t})+ \\
& \mathrm{q}_{43}^{(10)}(\mathrm{t}) \odot \mathrm{A}_{3}(\mathrm{t})+\mathrm{q}_{4,12}^{(10)}(\mathrm{t}) \odot \mathrm{A}_{12}(\mathrm{t}), \\
& \mathrm{A}_{5}(\mathrm{t})=\mathrm{M}_{5}(\mathrm{t})+\mathrm{q}_{50}(\mathrm{t}) \odot \mathrm{A}_{0}(\mathrm{t})+\mathrm{q}_{57}(\mathrm{t}) \odot \mathrm{A}_{7}(\mathrm{t})+ \\
& \mathrm{q}_{52}^{(11)}(\mathrm{t}) \odot \mathrm{A}_{2}(\mathrm{t})+\mathrm{q}_{5,13}^{(11)}(\mathrm{t}) \odot \mathrm{A}_{13}(\mathrm{t}) \text {, } \\
& \mathrm{A}_{6}(\mathrm{t})=\mathrm{M}_{6}(\mathrm{t})+\mathrm{q}_{61}(\mathrm{t}) \odot \mathrm{A}_{1}(\mathrm{t})+\mathrm{q}_{63}^{(12)}(\mathrm{t}) \odot \mathrm{A}_{3}(\mathrm{t}), \\
& \mathrm{A}_{7}(\mathrm{t})=\mathrm{M}_{7}(\mathrm{t})+\mathrm{q}_{70}(\mathrm{t}) \odot \mathrm{A}_{0}(\mathrm{t})+\mathrm{q}_{72}^{(13)}(\mathrm{t}) \odot \mathrm{A}_{2}(\mathrm{t}) \text {, } \\
& \mathrm{A}_{10}(\mathrm{t})=\mathrm{q}_{10,3}(\mathrm{t}) \odot \mathrm{A}_{3}(\mathrm{t})+\mathrm{q}_{10,12}(\mathrm{t}) \odot \mathrm{A}_{12}(\mathrm{t}), \\
& \mathrm{A}_{11}(\mathrm{t})=\mathrm{q}_{11,2}(\mathrm{t}) \odot \mathrm{A}_{2}(\mathrm{t})+\mathrm{q}_{11,13}(\mathrm{t}) \odot \mathrm{A}_{13}(\mathrm{t}), \\
& \mathrm{A}_{12}(\mathrm{t})=\mathrm{q}_{12,3}(\mathrm{t}) \odot \mathrm{A}_{3}(\mathrm{t}) \text {, } \\
& \mathrm{A}_{13}(\mathrm{t})=\mathrm{q}_{13,2}(\mathrm{t}) \odot \mathrm{A}_{2}(\mathrm{t})
\end{aligned}
$$

Where:

$$
\begin{aligned}
\mathrm{M}_{0}(\mathrm{t})=\overline{\mathrm{F}}_{1}(\mathrm{t}) \overline{\mathrm{L}}_{1}(\mathrm{t}), \mathrm{M}_{1}(\mathrm{t})=\overline{\mathrm{F}}_{2}(\mathrm{t}) \overline{\mathrm{L}}_{2}(\mathrm{t}), \mathrm{M}_{2}(\mathrm{t})= \\
\overline{\mathrm{F}}_{2}(\mathrm{t}) \overline{\mathrm{G}}_{1}(\mathrm{t}) \mathrm{M}_{3}(\mathrm{t})=\overline{\mathrm{F}}_{1}(\mathrm{t}) \overline{\mathrm{G}}_{2}(\mathrm{t}) \\
\mathrm{M}_{4}(\mathrm{t})=\overline{\mathrm{F}}_{2}(\mathrm{t}) \overline{\mathrm{H}}_{1}(\mathrm{t}), \mathrm{M}_{5}(\mathrm{t})=\overline{\mathrm{F}}_{1}(\mathrm{t}) \overline{\mathrm{H}}_{2}(\mathrm{t}), \mathrm{M}_{6}
\end{aligned}
$$$$
(\mathrm{t})=\overline{\mathrm{F}}_{2}(\mathrm{t}) \overline{\mathrm{K}}_{1}(\mathrm{t})=\overline{\mathrm{F}}_{1}(\mathrm{t}) \overline{\mathrm{K}}_{2}(\mathrm{t})
$$

Taking Laplace transforms of Eq. 1 and solving for $\mathrm{A}_{0}^{*}(\mathrm{~s})$, it gives:

$$
\mathrm{A}_{0}^{*}(\mathrm{~s})=\mathrm{N}_{1}(\mathrm{~s}) / \mathrm{D}_{1}(\mathrm{~s})
$$

Where:

$$
\begin{aligned}
\mathrm{N}_{1}(\mathrm{~s}) & =\mathrm{M}_{0}^{*}\left(1-\mathrm{b}_{23} \mathrm{~b}_{32}-\mathrm{q}_{23}^{*} \mathrm{~b}_{32} \mathrm{~b}_{21}\right)+\mathrm{M}_{1}^{*}\left[\mathrm{q}_{01}^{*}\left(1-\mathrm{b}_{23} \mathrm{~b}_{32}\right)+\left[\mathrm{q}_{02}^{*} \mathrm{~b}_{21}\right]\right. \\
& +\mathrm{q}_{01}^{*} \mathrm{q}_{13}^{*}\left(\mathrm{~b}_{32} \mathrm{~b}_{26}+\mathrm{b}_{37}\right)+\mathrm{q}_{02}^{*}\left[\mathrm{~b}_{26}+\mathrm{b}_{37}\left(\mathrm{~b}_{23}+\mathrm{b}_{21} \mathrm{q}_{13}^{*}\right)\right]
\end{aligned}
$$

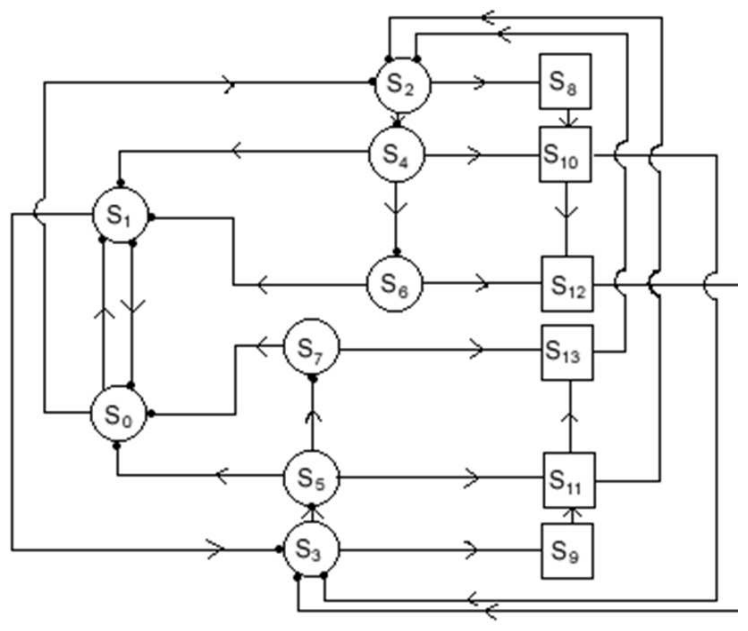

OUp state

Down state

- Regenerative point

Fig. 1: States and possible transitions and:

$$
\begin{aligned}
& \mathrm{D}_{1}(\mathrm{~s})=\left(1-\mathrm{b}_{23} \mathrm{~b}_{32}\right)\left(1-\mathrm{q}_{01}^{*} \mathrm{q}_{10}^{*} \mathrm{q}_{13}^{*}\left(\mathrm{~b}_{30} \mathrm{q}_{01}^{*}+\mathrm{b}_{32} \mathrm{~b}_{21}\right)\right. \\
& \quad-\mathrm{q}_{02}^{*}\left[\mathrm{~b}_{21}\left(\mathrm{q}_{10}^{*}+\mathrm{q}_{13}^{*} \mathrm{~b}_{30}\right)+\mathrm{b}_{23} \mathrm{~b}_{30}\right] \\
& \mathrm{b}_{21}=\mathrm{q}_{24}^{*}\left(\mathrm{q}_{41}^{*}+\mathrm{q}_{46}^{*} \mathrm{q}_{61}^{*}\right) \\
& \mathrm{b}_{23}= \mathrm{q}_{24}^{*}\left(\mathrm{q}_{46}^{*} \mathrm{q}_{63}^{(12)^{*}}+\mathrm{q}_{43}^{(10)^{*}}+\mathrm{q}_{4,12}^{(10)^{*}} \mathrm{q}_{12,3}^{*}\right)+\mathrm{q}_{2,10}^{(8)^{*}}\left(\mathrm{q}_{10,3}^{*}+\mathrm{q}_{10,12}^{*} \mathrm{q}_{12,3}^{*}\right), \\
& \mathrm{b}_{26}= \mathrm{M}_{2}^{*}+\mathrm{q}_{24}^{*}\left(\mathrm{M}_{4}^{*}+\mathrm{q}_{46}^{*} \mathrm{M}_{6}^{*}\right), \mathrm{b}_{30}=\mathrm{q}_{35}^{*}\left(\mathrm{q}_{50}^{*}+\mathrm{q}_{57}^{*} \mathrm{q}_{70}^{*}\right), \\
& \mathrm{b}_{32}=\mathrm{q}_{35}^{*}\left(\mathrm{q}_{57}^{*} \mathrm{q}_{72}^{(13)^{*}}+\mathrm{q}_{52}^{(11)^{*}}+\mathrm{q}_{5,13}^{(11)^{*}} \mathrm{q}_{13,2}^{*}\right)+\mathrm{q}_{3,11}^{(9) *}\left(\mathrm{q}_{11,2}^{*}+\mathrm{q}_{11,13}^{*} \mathrm{q}_{13,2}^{*}\right), \\
& \mathrm{b}_{37}=\mathrm{M}_{3}^{*}+\mathrm{q}_{35}^{*}\left(\mathrm{M}_{5}^{*}+\mathrm{q}_{57}^{*} \mathrm{M}_{7}^{*}\right)
\end{aligned}
$$

The steady state availability of the system is:

$\mathrm{A}_{0}(\infty)=\mathrm{N}_{1} / \mathrm{D}_{1}$

Where:

$$
\begin{aligned}
\mathrm{N}_{1}= & \mu_{0}\left(1-\overline{\mathrm{b}}_{23} \overline{\mathrm{b}}_{32}-\mathrm{p}_{13} \overline{\mathrm{b}}_{32} \overline{\mathrm{b}}_{21}\right)+\mu_{1}\left[\mathrm{p}_{01}\left(1-\overline{\mathrm{b}}_{23} \overline{\mathrm{b}}_{32}\right)+\mathrm{p}_{02} \overline{\mathrm{b}}_{21}\right] \\
& +\mathrm{p}_{01} \mathrm{p}_{13}\left(\overline{\mathrm{b}}_{23} \overline{\mathrm{b}}_{26}+\overline{\mathrm{b}}_{37}\right)+\mathrm{p}_{02}\left[\overline{\mathrm{b}}_{26}+\overline{\mathrm{b}}_{37}\left(\overline{\mathrm{b}}_{23}+\overline{\mathrm{b}}_{21} \mathrm{p}_{13}\right)\right]
\end{aligned}
$$

and:

$$
\begin{aligned}
& D_{1}=\left(1-\bar{b}_{23} \bar{b}_{32}\right)\left(\mu_{01} p_{10}+p_{01} \mu_{10}\right)-\left(\bar{b}_{23} \bar{b}_{32}^{\prime}+\bar{b}_{23}^{\prime} \bar{b}_{32}\right)\left(1-p_{02} p_{10}\right)+ \\
& \mu_{13}\left(\bar{b}_{30} p_{01}+\bar{b}_{32} \bar{b}_{21}\right)-p_{13}\left(\bar{b}_{30}^{\prime} p_{01}+\bar{b}_{30} \mu_{01}+\bar{b}_{32}^{\prime}+\bar{b}_{21}+\bar{b}_{32} \bar{b}^{\prime}{ }_{21}\right) \\
& +\mu_{02}\left[\bar{b}_{21}\left(p_{10}+p_{13} \bar{b}_{30}\right)+\bar{b}_{23} \bar{b}_{30}\right]-p_{02}\left[\bar{b}_{21}^{\prime}\left(p_{10}+p_{13} \bar{b}_{30}\right)\right. \\
& \left.-\bar{b}_{21}\left(\mu_{10}+\mu_{13} \bar{b}_{30}-p_{13} \bar{b}_{30}^{\prime}\right)+\bar{b}_{23}^{\prime} \bar{b}_{30}+\bar{b}_{23} \bar{b}_{30}^{\prime}\right] \text {, } \\
& \overline{\mathrm{b}}_{21}=\mathrm{p}_{24}\left(\mathrm{p}_{41}+\mathrm{p}_{46} \mathrm{p}_{61}\right) \text {, } \\
& \overline{\mathrm{b}}_{23}=\mathrm{p}_{24}\left(\mathrm{p}_{46} \mathrm{p}_{63}^{(12)}+\mathrm{p}_{43}^{(10)}+\mathrm{p}_{4,12}^{(10)} \mathrm{p}_{12,3}\right)+\mathrm{p}_{2,10}^{(8)}\left(\mathrm{p}_{10,3}+\mathrm{p}_{10,12} \mathrm{p}_{12,3}\right) \text {, } \\
& \overline{\mathrm{b}}_{26}=\mu_{2}+\mathrm{p}_{24}\left(\mu_{4}+\mathrm{p}_{46} \mu_{6}\right) \text {, } \\
& \overline{\mathrm{b}}_{30}=\mathrm{p}_{35}\left(\mathrm{p}_{50}+\mathrm{p}_{57} \mathrm{p}_{70}\right) \text {, } \\
& \overline{\mathrm{b}}_{32}=\mathrm{p}_{35}\left(\mathrm{p}_{57} \mathrm{p}_{72}^{(13)}+\mathrm{p}_{52}^{(11)}+\mathrm{p}_{5,13}^{(11)} \mathrm{p}_{13,2}\right)+\mathrm{p}_{3,11}^{(9)}\left(\mathrm{p}_{11,2}+\mathrm{p}_{11,13} \mathrm{p}_{13,2}\right), \\
& \overline{\mathrm{b}}_{37}=\mu_{3}+\mathrm{p}_{35}\left(\mu_{5}+\mathrm{p}_{57} \mu_{7}\right) \text {, } \\
& \overline{\mathrm{b}}_{21}^{\prime}=-\mu_{24}\left(\mathrm{p}_{41}+\mathrm{p}_{46} \mathrm{p}_{61}\right)-\mathrm{p}_{24}\left(\mu_{41}+\mu_{46} \mathrm{p}_{61}+\mathrm{p}_{46} \mu_{61}\right) \text {, } \\
& \overline{\mathrm{b}}_{23}^{\prime}=-\mu_{24}\left(\mathrm{p}_{46} \mathrm{p}_{63}^{(12)}+\mathrm{p}_{43}^{(10)}+\mathrm{p}_{4,12}^{(10)} \mathrm{p}_{12,3}\right)-\mathrm{p}_{24}\left(\mu_{46} \mathrm{p}_{63}^{(12)}\right. \\
& \left.+\mathrm{p}_{46} \mu_{63}^{(12)}+\mu_{43}^{(10)}+\mu_{4,12}^{(10)} \mathrm{p}_{12,3}+\mathrm{p}_{4,12}^{(10)} \mu_{12,3}\right) \mu_{2,10}^{(8)} \\
& +\left(\mathrm{p}_{10,3}+\mathrm{p}_{10,12} \mathrm{p}_{12,3}\right)-\mathrm{p}_{2,10}^{(8)}\left(\mu_{10,3}+\mu_{10,12} \mathrm{p}_{12,3}+\mathrm{p}_{10,12} \mu_{12,3}\right), \\
& \overline{\mathrm{b}}_{30}^{\prime}=-\mathrm{m}_{35}\left(\mathrm{p}_{50}+\mathrm{p}_{57} \mathrm{p}_{70}\right) \mathrm{p}_{35}\left(\mathrm{~m}_{50}+\mathrm{m}_{57} \mathrm{p}_{70}+\mathrm{p}_{57 \mathrm{~m} 70}\right) \text {, } \\
& \overline{\mathrm{b}}_{23}=\mathrm{p}_{24}\left(\mathrm{p}_{46} \mathrm{p}_{63}^{(12)}+\mathrm{p}_{43}^{(10)}+\mathrm{p}_{4,12}^{(10)} \mathrm{p}_{12,3}\right)+ \\
& \mathrm{p}_{2,10}^{(8)}\left(\mathrm{p}_{10,3}+\mathrm{p}_{10,12} \mathrm{p}_{12,3}\right) \text {, } \\
& \bar{b}_{32}^{\prime}=-\mu_{35}\left(p_{57} p_{72}^{(13)}+p_{52}^{(11)}+p_{5,13}^{(11)} p_{13,2}\right)-p_{35}\left(\mu_{57} p_{72}^{(13)}\right. \\
& \left.+\mathrm{p}_{57} \mu_{72}^{(13)}+\mu_{52}^{(11)}+\mu_{5,13}^{(11)} \mathrm{p}_{13,2}+\mathrm{p}_{5,13}^{(11)} \mu_{13,2}\right) \\
& -\mu_{3,11}^{(9)}\left(\mathrm{p}_{11,2}+\mathrm{p}_{11,13} \mathrm{p}_{13,2}\right)-\mathrm{p}_{3,11}^{(9)}\left(\mu_{11,2}+\mu_{11,13} \mathrm{p}_{13,2}\right. \\
& \left.+\mathrm{p}_{11,13} \mu_{13,2}\right)
\end{aligned}
$$


Busy period analysis: We define $B_{i}(t)$ is the probability that the system is under repair at epoch $t$ given that the system entered a regenerative state $S_{\mathrm{i}}$ at $\mathrm{t}=0$. By probabilistic arguments we have:

$$
\begin{aligned}
\mathrm{B}_{0}(\mathrm{t})= & \mathrm{q}_{01}(\mathrm{t}) \odot \mathrm{B}_{1}(\mathrm{t})+\mathrm{q}_{02}(\mathrm{t}) \odot \mathrm{B}_{2}(\mathrm{t}), \\
\mathrm{B}_{1}(\mathrm{t})= & \mathrm{q}_{10}(\mathrm{t}) \odot \mathrm{B}_{0}(\mathrm{t})+\mathrm{q}_{13}(\mathrm{t}) \odot \mathrm{B}_{3}(\mathrm{t}), \\
\mathrm{B}_{2}(\mathrm{t})= & \mathrm{V}_{2}(\mathrm{t})+\mathrm{q}_{24}(\mathrm{t}) \odot \mathrm{B}_{4}(\mathrm{t})+\mathrm{q}_{2,10}^{(8)}(\mathrm{t}) \odot \mathrm{B}_{10}(\mathrm{t}), \\
\mathrm{B}_{3}(\mathrm{t})= & \mathrm{V}_{3}(\mathrm{t})+\mathrm{q}_{35}(\mathrm{t}) \odot \mathrm{B}_{5}(\mathrm{t})+\mathrm{q}_{3,11}^{(9)}(\mathrm{t}) \odot \mathrm{B}_{11}(\mathrm{t}), \\
\mathrm{B}_{4}(\mathrm{t})= & \mathrm{V}_{4}(\mathrm{t})+\mathrm{q}_{11}(\mathrm{t}) \odot \mathrm{B}_{1}(\mathrm{t})+\mathrm{q}_{46}(\mathrm{t}) \odot \mathrm{B}_{6}(\mathrm{t})+ \\
& \mathrm{q}_{43}^{(1)}(\mathrm{t}) \odot \mathrm{B}_{3}(\mathrm{t})+\mathrm{q}_{4,12}^{(10)}(\mathrm{t}) \odot \mathrm{B}_{12}(\mathrm{t}), \\
\mathrm{B}_{5}(\mathrm{t})= & \mathrm{V}_{5}(\mathrm{t})+\mathrm{q}_{50}(\mathrm{t}) \odot \mathrm{B}_{0}(\mathrm{t})+\mathrm{q}_{57}(\mathrm{t}) \odot \mathrm{B}_{7}(\mathrm{t})+ \\
& \mathrm{q}_{52}^{(11)}(\mathrm{t}) \odot \mathrm{B}_{2}(\mathrm{t})+\mathrm{q}_{5,13}^{(1)}(\mathrm{t}) \odot \mathrm{B}_{13}(\mathrm{t}), \\
\mathrm{B}_{6}(\mathrm{t})= & \mathrm{V}_{6}(\mathrm{t})+\mathrm{q}_{61}(\mathrm{t}) \odot \mathrm{B}_{1}(\mathrm{t})+\mathrm{q}_{63}^{(12)}(\mathrm{t}) \odot \mathrm{B}_{3}(\mathrm{t}), \\
\mathrm{B}_{7}(\mathrm{t})= & \mathrm{V}_{7}(\mathrm{t})+\mathrm{q}_{70}(\mathrm{t}) \odot \mathrm{B}_{0}(\mathrm{t})+\mathrm{q}_{72}^{(13)}(\mathrm{t}) \odot \mathrm{B}_{2}(\mathrm{t}), \\
\mathrm{B}_{10}(\mathrm{t})= & \mathrm{V}_{10}(\mathrm{t})+\mathrm{q}_{10,3}(\mathrm{t}) \odot \mathrm{B}_{3}(\mathrm{t})+\mathrm{q}_{10,12}(\mathrm{t}) \odot \mathrm{B}_{12}(\mathrm{t}), \\
\mathrm{B}_{11}(\mathrm{t})= & \mathrm{V}_{11}(\mathrm{t})+\mathrm{q}_{11,2}(\mathrm{t}) \odot \mathrm{B}_{2}(\mathrm{t})+\mathrm{q}_{11,13}(\mathrm{t}) \odot \mathrm{B}_{13}(\mathrm{t}), \\
\mathrm{B}_{12}(\mathrm{t})= & \mathrm{V}_{12}(\mathrm{t})+\mathrm{q}_{12,3}(\mathrm{t}) \odot \mathrm{B}_{3}(\mathrm{t}), \\
\mathrm{B}_{13}(\mathrm{t})= & \mathrm{V}_{13}(\mathrm{t})+\mathrm{q}_{13,2}(\mathrm{t}) \odot \mathrm{B}_{2}(\mathrm{t})
\end{aligned}
$$

Where:

$$
\begin{aligned}
& \mathrm{V}_{2}(\mathrm{t})=\overline{\mathrm{F}}_{2}(\mathrm{t}) \overline{\mathrm{G}}_{1}(\mathrm{t}), \mathrm{V}_{3}(\mathrm{t})=\overline{\mathrm{F}}_{1}(\mathrm{t}) \overline{\mathrm{G}}_{2}(\mathrm{t}), \mathrm{V}_{4}(\mathrm{t}) \\
& =\overline{\mathrm{F}}_{2}(\mathrm{t}) \overline{\mathrm{H}}_{1}(\mathrm{t}), \mathrm{V}_{5}(\mathrm{t})=\overline{\mathrm{F}}_{1}(\mathrm{t}) \overline{\mathrm{H}}_{2}(\mathrm{t}) \\
& \mathrm{V}_{6}(\mathrm{t})=\overline{\mathrm{F}}_{2}(\mathrm{t}) \overline{\mathrm{K}}_{1}(\mathrm{t}), \mathrm{V}_{7}(\mathrm{t})=\overline{\mathrm{F}}_{1}(\mathrm{t}) \overline{\mathrm{K}}_{2}(\mathrm{t}), \\
& \mathrm{V}_{10}(\mathrm{t})=\overline{\mathrm{H}}_{1}(\mathrm{t}), \mathrm{V}_{11}(\mathrm{t})=\overline{\mathrm{H}}_{2}(\mathrm{t}) \\
& \mathrm{V}_{12}(\mathrm{t})=\overline{\mathrm{K}}_{1}(\mathrm{t}), \mathrm{V}_{13}(\mathrm{t})=\overline{\mathrm{K}}_{2}(\mathrm{t})
\end{aligned}
$$

Taking Laplace transforms of Eq. 5 and solving for $\mathrm{B}_{0}^{*}(\mathrm{~s})$, it follows:

$$
\mathrm{B}_{0}^{*}(\mathrm{~s})=\mathrm{N}_{2}(\mathrm{~s}) / \mathrm{D}_{1}(\mathrm{~s})
$$

Where:

$$
\begin{aligned}
\mathrm{N}_{2}(\mathrm{~s})= & \mathrm{q}_{01}^{*} \mathrm{q}_{13}^{*}\left(\mathrm{~b}_{2,12} \mathrm{~b}_{32}+\mathrm{b}_{3,12}\right)+\mathrm{q}_{02}^{*}\left[\mathrm{~b}_{2,12}+\mathrm{b}_{23} \mathrm{~b}_{3,13}\right. \\
& \left.+\mathrm{b}_{21} \mathrm{q}_{13}^{*} \mathrm{~b}_{3,13}\right]
\end{aligned}
$$

and:

$\mathrm{D}_{1}(\mathrm{~s}), \mathrm{b}_{21}, \mathrm{~b}_{23}, \mathrm{~b}_{25}, \mathrm{~b}_{30}, \mathrm{~b}_{32}, \mathrm{~b}_{37}$ are given in (4):

$$
\begin{aligned}
\mathrm{b}_{2,12}= & \mathrm{V}_{2}^{*}+\mathrm{q}_{2,10}^{(8) *}\left(\mathrm{~V}_{10}^{*}+\mathrm{q}_{10,12}^{*} \mathrm{~V}_{12}^{*}\right)+\mathrm{q}_{24}^{*}\left(\mathrm{~V}_{4}^{*}+\mathrm{q}_{46}^{*} \mathrm{~V}_{6}^{*}\right. \\
& \left.+\mathrm{q}_{4,12}^{(10) *} \mathrm{~V}_{12}^{*}\right) \\
\mathrm{b}_{3,13} & =\mathrm{V}_{1}^{*}+\mathrm{q}_{3,11}^{(9)}\left(\mathrm{V}_{11}^{*}+\mathrm{q}_{11,13}^{*} \mathrm{~V}_{13}^{*}\right)+\mathrm{q}_{35}^{*}\left(\mathrm{~V}_{5}^{*}+\mathrm{q}_{57}^{*} \mathrm{~V}_{7}^{*}\right. \\
& \left.+\mathrm{q}_{5,13}^{(11)^{*}} \mathrm{~V}_{13}^{*}\right)
\end{aligned}
$$

In long run the fraction of time for which the server is busy is given by:

$\mathrm{B}_{0}(\infty)=\mathrm{N}_{2} / \mathrm{D}_{1}$

Where:

$$
\begin{aligned}
\mathrm{N}_{2}= & \mathrm{p}_{01} \mathrm{p}_{13}\left(\overline{\mathrm{b}}_{2,12} \overline{\mathrm{b}}_{32}+\overline{\mathrm{b}}_{3,13}\right)+\mathrm{p}_{02}\left(\overline{\mathrm{b}}_{2,12}+\overline{\mathrm{b}}_{23} \overline{\mathrm{b}}_{3,13}\right. \\
& \left.+\overline{\mathrm{b}}_{21} \mathrm{p}_{13} \overline{\mathrm{b}}_{3,13}\right)
\end{aligned}
$$

and:

$\mathrm{D}_{1}, \overline{\mathrm{b}}_{21}, \overline{\mathrm{b}}_{23}, \overline{\mathrm{b}}_{26}, \overline{\mathrm{b}}_{30}, \overline{\mathrm{b}}_{32}, \overline{\mathrm{b}}_{37}, \overline{\mathrm{b}}_{21}^{\prime}, \overline{\mathrm{b}}_{23}^{\prime}, \overline{\mathrm{b}}_{30}^{\prime}, \overline{\mathrm{b}}_{32}^{\prime}$ are given in (4):

$$
\begin{aligned}
& \overline{\mathrm{b}}_{2,12}=\mu_{2}+\mathrm{p}_{2,10}^{(8)}\left(\mu_{10}+\mathrm{p}_{10,12} \mu_{12}\right)+\mathrm{p}_{24}\left(\mu_{4}+\mathrm{p}_{46} \mu_{6}+\mathrm{p}_{4,12}^{(10)} \mu_{12}\right), \\
& \overline{\mathrm{b}}_{3,13}=\mu_{3}+\mathrm{p}_{3,11}^{(9)}\left(\mu_{11}+\mathrm{p}_{11,13} \mu_{13}\right)+\mathrm{p}_{35}\left(\mu_{5}+\mathrm{p}_{57} \mu_{7}+\mathrm{p}_{5,13}^{(1)} \mu_{13}\right)
\end{aligned}
$$

The expected busy period of server in $(0,1]$ is:

$$
\mu_{\mathrm{b}}(\mathrm{t})=\int_{0}^{\mathrm{t}} \mathrm{B}_{0}(\mathrm{u}) \mathrm{du}
$$

So that:

$$
\mu_{\mathrm{b}}^{*}(\mathrm{~s})=\mathrm{B}_{0}^{*}(\mathrm{~s}) / \mathrm{s}
$$

Thus one can evaluate $\mu_{\mathrm{b}}(\mathrm{t})$ by taking inverse Laplace transform of $\mu_{\mathrm{b}}^{*}(\mathrm{~s})$.

Expected idle time of the repairman in $(0, t]$ is:

$$
\mu_{1}(t)=1-\mu_{b}(t)
$$

Expected number of visits by the repairman: According to the definition of $\mathrm{V}_{\mathrm{i}}(\mathrm{t})$ by elementary probability arguments, the following relations are obtained:

$$
\begin{aligned}
& \mathrm{V}_{0}(\mathrm{t})=\mathrm{Q}_{01}(\mathrm{t}) \mathrm{s} \mathrm{V}_{1}(\mathrm{t})+\mathrm{Q}_{02}(\mathrm{t}) \mathrm{s}\left[1+\mathrm{V}_{2}(\mathrm{t})\right], \mathrm{V}_{1}(\mathrm{t}) \\
& =\mathrm{Q}_{10}(\mathrm{t}) \mathrm{s} \mathrm{V}_{0}(\mathrm{t})+\mathrm{Q}_{13}(\mathrm{t}) \mathrm{s}\left[1+\mathrm{V}_{3}(\mathrm{t})\right] \text {, } \\
& \mathrm{V}_{2}(\mathrm{t})=\mathrm{Q}_{24}(\mathrm{t}) \mathrm{s} \mathrm{V}_{4}(\mathrm{t})+\mathrm{Q}_{2,10}^{(8)}(\mathrm{t}) \mathrm{s}_{10}(\mathrm{t}), \mathrm{V}_{3}(\mathrm{t})= \\
& \mathrm{Q}_{35}(\mathrm{t}) \mathrm{s} \mathrm{V}_{5}(\mathrm{t})+\mathrm{Q}_{3,11}^{(9)}(\mathrm{t}) \mathrm{s} \mathrm{V}_{11}(\mathrm{t}) \text {, } \\
& \mathrm{V}_{4}(\mathrm{t})=\mathrm{Q}_{41}(\mathrm{t}) \mathrm{s} \mathrm{V}_{1}(\mathrm{t})+\mathrm{Q}_{46}(\mathrm{t}) \mathrm{sV}_{6}(\mathrm{t})+\mathrm{Q}_{43}^{(10)}(\mathrm{t}) \mathrm{sV}_{3}(\mathrm{t}) \\
& +\mathrm{Q}_{4,12}^{(10)}(\mathrm{t}) \mathrm{sV}_{12}(\mathrm{t}) \\
& \mathrm{V}_{5}(\mathrm{t})=\mathrm{Q}_{50}(\mathrm{t}) \mathrm{sV}_{0}(\mathrm{t})+\mathrm{Q}_{57}(\mathrm{t}) \mathrm{sV}_{7}(\mathrm{t})+\mathrm{Q}_{52}^{(11)}(\mathrm{t}) \mathrm{sV}_{2} \\
& (\mathrm{t})+\mathrm{Q}_{5,13}^{(11)}(\mathrm{t}) \mathrm{sV}_{13}(\mathrm{t}) \text {, } \\
& \mathrm{V}_{6}(\mathrm{t})=\mathrm{Q}_{61}(\mathrm{t}) \mathrm{sV}_{1}(\mathrm{t})+\mathrm{Q}_{63}^{(12)}(\mathrm{t}) \mathrm{sV}_{3}(\mathrm{t}), \mathrm{V}_{7}(\mathrm{t}) \\
& =\mathrm{Q}_{70}(\mathrm{t}) \mathrm{sV}_{0}(\mathrm{t})+\mathrm{Q}_{72}^{(13)}(\mathrm{t}) \mathrm{sV}_{2}(\mathrm{t}) \text {, } \\
& \mathrm{V}_{10}(\mathrm{t})=\mathrm{Q}_{10,3}(\mathrm{t}) \mathrm{s} \mathrm{V}_{3}(\mathrm{t})+\mathrm{Q}_{10,12}(\mathrm{t}) \mathrm{s} \mathrm{V}_{12}(\mathrm{t}), \\
& \mathrm{V}_{11}(\mathrm{t})=\mathrm{Q}_{11,2}(\mathrm{t}) \mathrm{s} \mathrm{V}_{2}(\mathrm{t})+\mathrm{Q}_{11,13}(\mathrm{t}) \mathrm{s} \mathrm{V}_{13}(\mathrm{t}), \\
& \mathrm{V}_{12}(\mathrm{t})=\mathrm{Q}_{12,3}(\mathrm{t}) \mathrm{s} \mathrm{V}_{3}(\mathrm{t}), \mathrm{V}_{13}(\mathrm{t})=\mathrm{Q}_{13,2}(\mathrm{t}) \mathrm{s} \mathrm{V}_{2}(\mathrm{t})
\end{aligned}
$$


Taking Laplace-Stieltjes transforms of Eq. 10 and solving for $\tilde{\mathrm{V}}_{0}(\mathrm{~s})$, dropping the argument "s" for brevity, it follows:

$$
\tilde{\mathrm{V}}_{0}(\mathrm{~s})=\mathrm{N}_{3}(\mathrm{~s}) / \mathrm{D}_{2}(\mathrm{~s})
$$

Where:

$$
\mathrm{N}_{3}(\mathrm{~s})=\left(\tilde{\mathrm{Q}}_{01} \tilde{\mathrm{Q}}_{13}+\tilde{\mathrm{Q}}_{02}\right)\left(1-\mathrm{C}_{23} \mathrm{C}_{32}\right)+\tilde{\mathrm{Q}}_{02} \mathrm{C}_{21} \tilde{\mathrm{Q}}_{13}\left(1-\mathrm{C}_{32}\right)
$$

and:

$$
\begin{aligned}
& \mathrm{D}_{2}(\mathrm{~s})=\left(1-\tilde{\mathrm{Q}}_{01} \tilde{\mathrm{Q}}_{10}\right)\left(1-\mathrm{C}_{23} \mathrm{C}_{30}\right)-\tilde{\mathrm{Q}}_{13}\left(\mathrm{C}_{30} \tilde{\mathrm{Q}}_{01}+\mathrm{C}_{32} \mathrm{C}_{21}\right)-\tilde{\mathrm{Q}}_{02}\left[\mathrm{C}_{21}\left(\tilde{\mathrm{Q}}_{10}+\tilde{\mathrm{Q}}_{13} \mathrm{C}_{30}\right)+\mathrm{C}_{23} \mathrm{C}_{30}\right] \\
& \mathrm{C}_{21}=\tilde{\mathrm{Q}}_{24}\left(\tilde{\mathrm{Q}}_{41}+\tilde{\mathrm{Q}}_{46} \tilde{\mathrm{Q}}_{61}\right), \\
& \mathrm{C}_{23}=\tilde{\mathrm{Q}}_{24}\left(\tilde{\mathrm{Q}}_{46} \tilde{\mathrm{Q}}_{63}^{(12)}+\tilde{\mathrm{Q}}_{43}^{(10)}+\tilde{\mathrm{Q}}_{4,12}^{(10)} \tilde{\mathrm{Q}}_{12,3}\right)+\tilde{\mathrm{Q}}_{2,10}^{(8)}\left(\tilde{\mathrm{Q}}_{10,3}+\tilde{\mathrm{Q}}_{10,12} \tilde{\mathrm{Q}}_{12,3}\right), \\
& \mathrm{C}_{30}=\tilde{\mathrm{Q}}_{35}\left(\tilde{\mathrm{Q}}_{50}+\tilde{\mathrm{Q}}_{57} \tilde{\mathrm{Q}}_{70}\right) \\
& \mathrm{C}_{32}=\tilde{\mathrm{Q}}_{35}\left(\tilde{\mathrm{Q}}_{57} \tilde{\mathrm{Q}}_{72}^{(13)}+\tilde{\mathrm{Q}}_{52}^{(11)}+\tilde{\mathrm{Q}}_{5,13}^{(1) 1} \tilde{\mathrm{Q}}_{13,2}\right)+\tilde{\mathrm{Q}}_{3,11}^{(9)}\left(\tilde{\mathrm{Q}}_{11,2}+\tilde{\mathrm{Q}}_{11,13} \tilde{\mathrm{Q}}_{13,2}\right)
\end{aligned}
$$

In steady state, number of visits per unit is given by:

$\mathrm{V}_{0}(\infty)=\mathrm{N}_{3} / \mathrm{D}_{2}$

Where:

$$
\mathrm{N}_{3}=\left(\mathrm{p}_{01} \mathrm{p}_{13}+\mathrm{p}_{02}\right)\left(1-\overline{\mathrm{C}}_{23} \overline{\mathrm{C}}_{32}\right)+\mathrm{p}_{02} \overline{\mathrm{C}}_{21} \mathrm{p}_{13}\left(1-\overline{\mathrm{C}}_{32}\right)
$$

and:

$$
\begin{aligned}
& \mathrm{D}_{2}=\left(\mathrm{m}_{01} \mathrm{p}_{10}+\mathrm{p}_{01} \mathrm{~m}_{10}\right)\left(1-\overline{\mathrm{C}}_{23} \overline{\mathrm{C}}_{30}\right)\left(1-\mathrm{t} \mathrm{p}_{01} \mathrm{p}_{10}\right)\left(\overline{\mathrm{C}}_{23}^{\prime} \overline{\mathrm{C}}_{30}+\overline{\mathrm{C}}_{23} \overline{\mathrm{C}}_{30}^{\prime}\right)+\mu_{13}\left(\overline{\mathrm{C}}_{30} \mathrm{p}_{01}+\overline{\mathrm{C}}_{32} \overline{\mathrm{C}}_{21}\right)+\mathrm{p}_{13}\left(-\overline{\mathrm{C}}_{30}^{\prime} \mathrm{p}_{01}+\overline{\mathrm{C}}_{30} \mu_{01}\right. \\
& \left.\left.-\overline{\mathrm{C}}_{32}^{\prime} \overline{\mathrm{C}}_{21}-\overline{\mathrm{C}}_{32} \overline{\mathrm{C}}_{21}^{\prime}\right)+\mu_{02} \overline{\mathrm{C}}_{21}\left(\mathrm{p}_{10}+\mathrm{p}_{13} \overline{\mathrm{C}}_{30}\right)+\overline{\mathrm{C}}_{23} \overline{\mathrm{C}}_{30}\right]+\mathrm{p}_{02}\left[-\overline{\mathrm{C}}_{21}^{\prime}\left(\mathrm{p}_{10}+\mathrm{p}_{13} \overline{\mathrm{C}}_{30}\right)+\overline{\mathrm{C}}_{21}\left(\mu_{10}+\mu_{13} \overline{\mathrm{C}}_{30}-\mathrm{p}_{13} \overline{\mathrm{C}}_{30}^{\prime}\right)\right. \\
& -\overline{\mathrm{C}}_{23}^{\prime} \overline{\mathrm{C}}_{30}-\overline{\mathrm{C}}_{23} \overline{\mathrm{C}}_{30}^{\prime} \text {, } \\
& \overline{\mathrm{C}}_{21}=\mathrm{p}_{24}\left(\mathrm{p}_{41}+\mathrm{p}_{46} \mathrm{p}_{61}\right) \text {, } \\
& \overline{\mathrm{C}}_{23}=\mathrm{p}_{24}\left(\mathrm{p}_{46} \mathrm{p}_{63}^{(12)}+\mathrm{p}_{43}^{(10)}+\mathrm{p}_{4,12}^{(10)} \mathrm{p}_{12,3}\right)+\mathrm{p}_{2,10}^{(8)}\left(\mathrm{p}_{10,3}+\mathrm{p}_{10,12} \mathrm{p}_{12,3}\right) \text {, } \\
& \overline{\mathrm{C}}_{30}=\mathrm{p}_{35}\left(\mathrm{p}_{50}+\mathrm{p}_{57} \mathrm{p}_{70}\right) \text {, } \\
& \overline{\mathrm{C}}_{32}=\mathrm{p}_{35}\left(\mathrm{p}_{57} \mathrm{p}_{72}^{(13)}+\mathrm{p}_{52}^{(11)}+\mathrm{p}_{5,13}^{(11)} \mathrm{p}_{13,2}\right)+\mathrm{p}_{3,11}^{(9)}\left(\mathrm{p}_{11,2}+\mathrm{p}_{11,13} \mathrm{p}_{13,2}\right) \\
& \overline{\mathrm{C}}_{21}^{\prime}=-\mathrm{m}_{02}\left(\mathrm{p}_{41}+\mathrm{p}_{46} \mathrm{p}_{61}\right)-\mathrm{p}_{24}\left(\mathrm{~m}_{41}+\mathrm{m}_{46} \mathrm{p}_{61}+\mathrm{p}_{46} \mathrm{~m}_{61}\right) \text {, } \\
& \overline{\mathrm{C}}_{23}^{\prime}=-\mu_{24}\left(\mathrm{p}_{46} \mathrm{p}_{63}^{(12)}+\mathrm{p}_{43}^{(10)}+\mathrm{p}_{4,12}^{(10)} \mathrm{p}_{12,3}\right)-\mathrm{p}_{24}\left(\mu_{46} \mathrm{p}_{63}^{(12)}+\mathrm{p}_{46} \mu_{63}^{(12)}+\mu_{43}^{(10)}+\mu_{4,12}^{(10)} \mathrm{p}_{12,3}+\mathrm{p}_{4,12}^{(10)} \mu_{12,3}\right)-\mu_{2,10}^{(8)}\left(\mathrm{p}_{10,3}+\mathrm{p}_{10,12} \mathrm{p}_{12,3}\right) \\
& -p_{2,10}^{(8)}\left(\mu_{10,3}+\mu_{10,12} p_{12,3}+p_{10,12} \mu_{12,3}\right) \text {, } \\
& \overline{\mathrm{C}}_{30}^{\prime}=-\mathrm{m}_{35}\left(\mathrm{p}_{50}+\mathrm{p}_{57} \mathrm{p}_{70}\right)-\mathrm{p}_{35}\left(\mathrm{~m}_{50}+\mathrm{m}_{57} \mathrm{p}_{70}+\mathrm{p}_{57} \mathrm{~m}_{70}\right) \text {, } \\
& \overline{\mathrm{C}}_{32}^{\prime}=-\mu_{35}\left(\mathrm{p}_{57} \mathrm{p}_{72}^{(13)}+\mathrm{p}_{52}^{(11)}+\mathrm{p}_{5,13}^{(11)} \mathrm{p}_{13,2}\right)-\mathrm{p}_{35}\left(\mu_{57} \mathrm{p}_{72}^{(13)}+\mathrm{p}_{57} \mu_{72}^{(13)}+\mu_{52}^{(11)}+\mu_{5,13}^{(11)} \mathrm{p}_{13,2}+\mathrm{p}_{5,13}^{(11)} \mu_{13,2}\right)-\mu_{3,11}^{(9)}\left(\mathrm{p}_{11,2}+\mathrm{p}_{11,13} \mathrm{p}_{13,2}\right) \\
& -p_{3,11}^{(9)}\left(\mu_{11,2}+\mu_{11,13} p_{13,2}+p_{11,13} \mu_{13,2}\right)
\end{aligned}
$$

Cost analysis: The cost function of the system obtained by considering the mean-up time of the system, expected busy period of the server and the expected number of visits by the server, therefore, the expected cost incurred in $(0$, t] is: 
$C(t)=$ expected total revenue in $(0, t] t$ expected total service cost in $(0, \mathrm{t}]$

- expected costof visits by server in $(0, t]=$ $\mathrm{K}_{1} \mathrm{~m}_{\text {up }}(\mathrm{t})-\mathrm{K}_{2} \mathrm{~m}_{\mathrm{b}}(\mathrm{t})-\mathrm{K}_{3} \mathrm{~V}_{0}(\mathrm{t})$

The expected profit per unit time in steady-state is:

$\mathrm{C}=\mathrm{K}_{1} \mathrm{~A}_{0}-\mathrm{K}_{2} \mathrm{~B}_{0}-\mathrm{K}_{3} \mathrm{~V}_{0}$

Where:

$\mathrm{K}_{1}=$ The revenue per unit up time

$\mathrm{K}_{2}=$ The cost per unit time for which system is under repair

$\mathrm{K}_{3}=$ The cost per visit by repair facility

Transition probabilities are:

$$
\begin{aligned}
& \mathrm{p}_{02}=\alpha_{1} /\left(\alpha_{1}+\beta_{1}\right), \\
& \mathrm{p}_{24}=\gamma_{1} /\left(\alpha_{2}+{ }_{1}\right), \\
& \mathrm{p}_{39}=\mathrm{p}_{3,11}^{(9)}=\alpha_{1} /\left(\alpha_{1}+\gamma_{2}\right), \\
& \mathrm{p}_{4,10}=\alpha_{2} /\left(\lambda_{1}+\alpha_{2}\right), \\
& \mathrm{p}_{5,11}=\alpha_{1} /\left(\alpha_{1}+\theta_{2}\right), \\
& \mathrm{p}_{70}=\lambda_{2} /\left(\alpha_{1}+\lambda_{2}\right), \\
& \mathrm{p}_{10,12}=\mathrm{q}_{1}, \\
& \mathrm{p}_{43}^{(10)}=\mathrm{p}_{1} \alpha_{2} /\left(\alpha_{2}+\theta_{1}\right), \\
& \mathrm{p}_{5,13}^{(11)}=\mathrm{q}_{2} \alpha_{1} /\left(\alpha_{1}+\theta_{2}\right),
\end{aligned}
$$

Special cases: The two units are dissimilar with exponential distributions:

Let:

$\alpha_{i} \quad$ Failure rate of the $i$-th operative; $i=1,2$

$\beta_{\mathrm{i}} \quad$ Rate of changes of the $\mathrm{i}$-th operative unit; $\mathrm{i}=1,2$

$\gamma_{i} \quad$ Rate of repair of the $i$-th failed unit; $i=1,2$

$\theta_{\mathrm{i}} \quad$ Rate of complete inspection of the $\mathrm{i}$-th failed unit; i = 1, 2

$\lambda_{\mathrm{i}} \quad$ Rate of complete post repair of the $\mathrm{i}$-th unsatisfactory failed unit; $i=1,2$,

$\mathrm{p}_{\mathrm{i}}=-\left(\mathrm{q}_{\mathrm{i}}\right)$ Probability that the repair of the $\mathrm{i}-\mathrm{th}$ unit is satisfactory after the inspection; $i=1,2$

$$
\begin{aligned}
& \mathrm{p}_{10}=\beta_{2} /\left(\alpha_{2}+\beta_{2}\right), \\
& \mathrm{p}_{28}=\mathrm{p}_{2,10}^{(8)}=\beta_{2} /\left(\alpha_{2}+\gamma_{1}\right), \\
& \mathrm{p}_{41}=\mathrm{p}_{1} \theta_{1} /\left(\alpha_{2}+\theta_{1}\right), \\
& \mathrm{p}_{50}=\mathrm{p}_{2} \theta_{2} /\left(\alpha_{1}+\theta_{2}\right), \\
& \mathrm{p}_{61}=\lambda_{1} /\left(\alpha_{2}+\lambda_{1}\right), \\
& \mathrm{p}_{7,13}=\mathrm{p}_{72}^{(13)}=\alpha_{1} /\left(\alpha_{1}+\lambda_{2}\right), \\
& \mathrm{p}_{11,2}=\mathrm{p}_{2}, \\
& \mathrm{p}_{4,12}^{(10)}=\mathrm{q}_{1} \alpha_{2} /\left(\alpha_{2}+\theta_{1}\right), \\
& \mathrm{p}_{8,10}=\mathrm{p}_{9,11}=\mathrm{p}_{12,3}=\mathrm{p}_{13,2}=1
\end{aligned}
$$

The mean sojourn times are:

$$
\begin{array}{lll}
\mu_{0}=1 /\left(\alpha_{1}+\beta_{1}\right), & \mu_{1}=1 /\left(\alpha_{2}+\beta_{2}\right), \mu_{2}=1 /\left(\alpha_{2}+\beta_{1}\right), & \mu_{3}=1 /\left(\alpha_{1}+\gamma_{2}\right), \\
\mu_{4}=1 /\left(\alpha_{2}+\theta_{1}\right), & \mu_{5}=1 /\left(\alpha_{1}+\theta_{2}\right), \mu_{6}=1 /\left(\alpha_{2}+\lambda_{1}\right), & \mu_{7}=1 /\left(\alpha_{1}+\lambda_{2}\right), \\
\mu_{10}=1 / \theta_{1}, & \mu_{11}=1 / \theta_{2}, \mu_{12}=1 / \lambda_{1}, & \mu_{13}=1 / \lambda_{2}
\end{array}
$$

The mean time to system failure with starting state $S_{0}$ is:

Where:

$$
\operatorname{MTSF}=\hat{\mathrm{N}}_{0} / \hat{\mathrm{D}}_{0}
$$

$$
\begin{aligned}
\hat{\mathrm{N}}_{0}= & \frac{1}{\left(\alpha_{1}+\beta_{1}\right)\left(\alpha_{2}+\beta_{2}\right)}\left\{\left[\frac{\left(\beta_{2}+\alpha_{2} \hat{\overline{\mathrm{a}}}_{30}\right)}{\left(\alpha_{2}+\beta_{2}\right)}-\alpha_{2} \hat{\overline{\mathrm{a}}}_{30}^{\prime}\right]\left(\beta_{1}+\alpha_{1} \hat{\overline{\mathrm{a}}}_{21}\right)+\left[\frac{\left(\beta_{1}+\alpha_{1} \hat{\overline{\mathrm{a}}}_{21}\right.}{\left(\alpha_{1}+\beta_{1}\right)}-\alpha_{1} \hat{\overline{\mathrm{a}}}_{21}^{\prime}\right]\left(\beta_{2}+\alpha_{2} \hat{\overline{\mathrm{a}}}_{30}\right)\right\}+\frac{\beta_{1} \alpha_{2}}{\left(\alpha_{1}+\beta_{1}\right)\left(\alpha_{2}+\beta_{2}\right)} \\
& \left\{\left[\frac{1}{\left(\alpha_{1}+\beta_{1}\right)}+\frac{1}{\left(\alpha_{2}+\beta_{2}\right)}\right] \hat{\overline{\mathrm{a}}}_{39}-\hat{\overline{\mathrm{a}}}_{39}^{\prime}\right\}+\frac{\alpha_{1}}{\left(\alpha_{1}+\beta_{1}\right)}\left[\hat{\overline{\mathrm{a}}}_{28}+\frac{\alpha_{2}}{\left(\alpha_{2}+\beta_{2}\right)} \hat{\overline{\mathrm{a}}}_{21} \hat{\overline{\mathrm{a}}}_{39}\right]-\frac{\alpha_{1}}{\left(\alpha_{1}+\beta_{1}\right)}\left[\hat{\overline{\mathrm{a}}}_{28}^{\prime}-\frac{\alpha_{2}}{\left(\alpha_{2}+\beta_{2}\right)} \hat{\overline{\mathrm{a}}}_{21} \hat{\overline{\mathrm{a}}}_{39}\right. \\
& \left.+\frac{\alpha_{2}}{\left(\alpha_{2}+\beta_{2}\right)}\left(\hat{\overline{\mathrm{a}}}_{28}^{\prime} \hat{\overline{\mathrm{a}}}_{39}+\hat{\overline{\mathrm{a}}}_{28} \hat{\overline{\mathrm{a}}}^{\prime}{ }_{39}\right)\right]
\end{aligned}
$$

and: 


$$
\begin{aligned}
& \hat{\mathrm{D}}_{0}=\frac{\left(\beta_{1}+\alpha_{1} \hat{\overline{\mathrm{a}}}_{21}\right)\left(\beta_{2} \alpha_{2} \hat{\overline{\mathrm{a}}}_{30}\right)}{\left(\alpha_{1}+\beta_{1}\right)\left(\alpha_{1}+\beta_{2}\right)} \hat{\overline{\mathrm{a}}}_{30}=\frac{\gamma_{2} \theta_{2}}{\left(\alpha_{1}+\gamma_{2}\right)\left(\alpha_{1}+\theta_{2}\right)}\left[\mathrm{p}_{2}+\frac{\mathrm{q}_{2} \lambda_{2}}{\left(\alpha_{1}+\lambda_{2}\right)}\right] \\
& \hat{\bar{a}}_{21}=\frac{\gamma_{1} \theta_{1}}{\left(\alpha_{2}+\gamma_{1}\right)\left(\alpha_{2}+\theta_{1}\right)}\left[p_{1}+\frac{\mathrm{q}_{1} \lambda_{1}}{\left(\alpha_{2}+\lambda_{1}\right)}\right] \hat{\overline{\mathrm{a}}}_{28}=\frac{\alpha_{2}}{\left(\alpha_{2}+\gamma_{1}\right)}\left\{1+\frac{\lambda_{1}}{\left(\alpha_{2}+\lambda_{1}\right)}\left[1+\frac{\mathrm{q}_{1} \theta_{1}}{\left(\alpha_{2}+\theta_{1}\right)}\right]\right\} \\
& \hat{\overline{\mathrm{a}}}_{39}=\frac{\alpha_{1}}{\left(\alpha_{1}+\gamma_{2}\right)}\left\{1+\frac{\lambda_{2}}{\left(\alpha_{1}+\lambda_{2}\right)}\left[1+\frac{\mathrm{q}_{2} \theta_{2}}{\left(\alpha_{1}+\theta_{2}\right)}\right]\right\} \\
& \hat{\overline{\mathrm{a}}}_{30}^{\prime}=\frac{\gamma_{2} \theta_{2}}{\left(\alpha_{1}+\gamma_{2}\right)\left(\alpha_{1}+\theta_{2}\right)}\left\{\frac{1}{\left(\alpha_{1}+\gamma_{2}\right)}\left[\mathrm{p}_{2}+\frac{\mathrm{q}_{2} \lambda_{2}}{\left(\alpha_{1}+\gamma_{2}\right)}\right]+\frac{\mathrm{p}_{2}}{\left(\alpha_{1}+\theta_{2}\right)}+\frac{\mathrm{q}_{2} \lambda_{2}}{\left(\alpha_{1}+\lambda_{2}\right)}\left[\frac{1}{\left(\alpha_{1}+\theta_{2}\right)}+\frac{1}{\left(\alpha_{1}+\lambda_{2}\right)}\right]\right\} \\
& \hat{\overline{\mathrm{a}}}_{21}^{\prime}=\frac{\gamma_{1} \theta_{1}}{\left(\alpha_{2}+\gamma_{1}\right)\left(\alpha_{2}+\theta_{1}\right)}\left\{\frac{1}{\left(\alpha_{2}+\gamma_{1}\right)}\left[\mathrm{p}_{1}+\frac{\mathrm{q}_{1} \lambda_{1}}{\left(\alpha_{2}+\gamma_{1}\right)}\right]+\frac{\mathrm{p}_{1}}{\left(\alpha_{2}+\theta_{1}\right)}+\frac{\mathrm{q}_{1} \lambda_{1}}{\left(\alpha_{2}+\lambda_{1}\right)}\left[\frac{1}{\left(\alpha_{2}+\theta_{1}\right)}+\frac{1}{\left(\alpha_{2}+\lambda_{1}\right)}\right]\right\} \\
& \hat{\overline{\mathrm{a}}}_{28}^{\prime}=\frac{\alpha_{2}}{\left(\alpha_{2}+\gamma_{1}\right)^{2}}-\frac{\gamma_{1} \alpha_{2}}{\left(\alpha_{2}+\gamma_{1}\right)\left(\alpha_{2}+\lambda_{1}\right)}\left\{\frac{1}{\left(\alpha_{2}+\gamma_{1}\right)}\left[1+\frac{\mathrm{q}_{1} \theta_{1}}{\left(\alpha_{2}+\theta_{1}\right)}\right]+\frac{\mathrm{q}_{1} \theta_{1}}{\left(\alpha_{2}+\theta_{1}\right)}\left[\frac{1}{\left(\alpha_{2}+\theta_{1}\right)}+\frac{1}{\left(\alpha_{2}+\lambda_{1}\right)}\right]-\frac{1}{\left(\alpha_{2}+\lambda_{1}\right)}\right\} \\
& \hat{\bar{a}}_{39}^{\prime}=\frac{\alpha_{1}}{\left(\alpha_{1}+\gamma_{2}\right)^{2}}-\frac{\gamma_{2} \alpha_{1}}{\left(\alpha_{1}+\gamma_{2}\right)\left(\alpha_{1}+\lambda_{2}\right)}\left\{\frac{1}{\left(\alpha_{1}+\gamma_{2}\right)}\left[1+\frac{\mathrm{q}_{2} \theta_{2}}{\left(\alpha_{1}+\theta_{2}\right)}\right]+\frac{\mathrm{q}_{2} \theta_{2}}{\left(\alpha_{1}+\theta_{2}\right)}\left[\frac{1}{\left(\alpha_{1}+\theta_{2}\right)}+\frac{1}{\left(\alpha_{1}+\lambda_{2}\right)}\right]-\frac{1}{\left(\alpha_{1}+\lambda_{2}\right)}\right\}
\end{aligned}
$$

In this case, $\hat{\mathrm{M}}_{\mathrm{i}}(\mathrm{t})$ are:

$$
\begin{array}{llll}
\hat{M}_{0}(t)=e^{-\left(\alpha_{1}+\beta_{1}\right) t}, & \hat{M}_{1}(t)=\mathrm{e}^{-\left(\alpha_{2}+\beta_{2}\right) t}, & \hat{M}_{0}(t)=e^{-\left(\alpha_{1}+\beta_{1}\right) t}, & \hat{M}_{3}(t)=e^{-\left(\alpha_{1}+\gamma_{2}\right) t} \\
\hat{M}_{4}(t)=e^{-\left(\alpha_{2}+\theta_{1}\right) t}, & \hat{M}_{5}(t)=e^{-\left(\alpha_{1}+\theta_{2}\right) t}, & \hat{M}_{6}(t)=e^{-\left(\alpha_{2}+\lambda_{1}\right) t}, & \hat{M}_{7}(t)=e^{-\left(\alpha_{1}+\alpha_{2}\right) t}
\end{array}
$$

The steady state availability of the system is:

$$
\hat{\mathrm{A}}_{0}(\infty)=\hat{\mathrm{N}}_{1} / \hat{\mathrm{D}}_{1}
$$

Where:

$$
\begin{aligned}
& \hat{\mathrm{N}}_{1}=\frac{1}{\left(\alpha_{1}+\beta_{1}\right)}\left\{\left[1-\hat{\overline{\mathrm{b}}}_{23} \hat{\overline{\mathrm{b}}}_{32}-\frac{\alpha_{2}}{\left(\alpha_{2}+\beta_{2}\right)} \hat{\overline{\mathrm{b}}}_{32} \hat{\mathrm{b}}_{21}\right]+\frac{1}{\left(\alpha_{2}+\beta_{2}\right)}\left[\beta_{1}\left(1-\hat{\overline{\mathrm{b}}}_{23} \hat{\overline{\mathrm{b}}}_{32}\right)+\alpha_{1} \hat{\overline{\mathrm{b}}}_{21}\right]+\alpha_{1}\left[\hat{\overline{\mathrm{b}}}_{26}+\hat{\overline{\mathrm{b}}}_{37}\left(\hat{\overline{\mathrm{b}}}_{23}+\frac{\alpha_{2}}{\left(\alpha_{2}+\beta_{2}\right)} \hat{\overline{\mathrm{b}}}_{21}\right)\right]\right\} \\
& \hat{D}_{1}=\frac{\beta_{1} \beta_{2}\left(1-\hat{\bar{b}}_{23} \hat{\bar{b}}_{32}\right)}{\left(\alpha_{1}+\beta_{1}\right)\left(\alpha_{2}+\beta_{2}\right)}\left[\frac{1}{\left(\alpha_{1}+\beta_{1}\right)}+\frac{1}{\left(\alpha_{2}+\beta_{2}\right)}\right]-\left(\hat{\bar{b}}_{23} \hat{\bar{b}}_{32}^{\prime}+\hat{\bar{b}}_{23}^{\prime} \hat{\bar{b}}_{32}\right)\left[1-\frac{\beta_{1} \beta_{2}}{\left(\alpha_{1}+\beta_{1}\right)\left(\alpha_{2}+\beta_{2}\right)}\right]+\frac{\alpha_{2}}{\left(\alpha_{2}+\beta_{2}\right)} \\
& \left\{\frac{\hat{\bar{b}}_{30} \beta_{1}}{\left(\alpha_{2}+\beta_{2}\right)\left(\alpha_{1}+\beta_{1}\right)}+\hat{\bar{b}}_{32} \hat{\bar{b}}_{21}-\left[\frac{\beta_{1}}{\left(\alpha_{1}+\beta_{1}\right)}\left(\hat{\overline{\mathrm{b}}}_{30}^{\prime}+\frac{\hat{\overline{\mathrm{b}}}_{30}}{\left(\alpha_{1}+\beta_{1}\right)}\right)+\hat{\overline{\mathrm{b}}}_{32}^{\prime} \hat{\overline{\mathrm{b}}}_{21}+\hat{\overline{\mathrm{b}}}_{32} \hat{\overline{\mathrm{b}}}_{21}^{\prime}\right]\right\}+\frac{\alpha_{1}}{\left(\alpha_{1}+\beta_{1}\right)}\left\{\frac{1}{\left(\alpha_{1}+\beta_{1}\right)}\left[\frac{\hat{\overline{\mathrm{b}}}_{21}}{\left(\alpha_{2}+\beta_{2}\right)}\left(\beta_{2}+\alpha_{2} \hat{\overline{\mathrm{b}}}_{30}\right)+\hat{\overline{\mathrm{b}}}_{23} \hat{\overline{\mathrm{b}}}_{30}\right]\right. \\
& \left.-\frac{\hat{\overline{\mathrm{b}}}_{21}^{\prime}}{\left(\alpha_{2}+\beta_{2}\right)}\left(\beta_{2}+\alpha_{2} \hat{\overline{\mathrm{b}}}_{30}\right)+\frac{\hat{\overline{\mathrm{b}}}_{21}}{\left(\alpha_{2}+\beta_{2}\right)}\left[\frac{\beta_{2}}{\left(\alpha_{2}+\beta_{2}\right)}+\frac{\alpha_{2} \hat{\overline{\mathrm{b}}}_{30}}{\left(\alpha_{2}+\beta_{2}\right)}-\alpha_{2} \hat{\overline{\mathrm{b}}}_{30}^{\prime}\right]-\hat{\overline{\mathrm{b}}}_{23}^{\prime} \hat{\overline{\mathrm{b}}}_{30}^{\prime}-\hat{\overline{\mathrm{b}}}_{23} \hat{\overline{\mathrm{b}}}_{30}^{\prime}\right\}, \\
& \hat{\overline{\mathrm{b}}}_{21}=\frac{\gamma_{1} \theta_{1}}{\left(\alpha_{2}+\gamma_{1}\right)\left(\alpha_{2}+\theta_{1}\right)}\left[\mathrm{p}_{1}+\frac{\mathrm{q}_{1} \lambda_{1}}{\left(\alpha_{2}+\lambda_{1}\right)}\right], \hat{\overline{\mathrm{b}}}_{23}=\frac{1}{\left(\alpha_{2}+\gamma_{1}\right)}\left\{\frac{\gamma_{1} \alpha_{2}}{\left(\alpha_{2}+\theta_{1}\right)}\left[\frac{\mathrm{q}_{1} \theta_{1}}{\left(\alpha_{2}+\lambda_{1}\right)}+1\right]+\alpha_{2}\right\} \text {, } \\
& \hat{\bar{b}}_{26}=\frac{1}{\left(\alpha_{2}+\gamma_{1}\right)}\left\{1+\frac{\gamma_{1}}{\left(\alpha_{2}+\theta_{1}\right)}\left[1+\frac{\mathrm{q}_{1} \theta_{1}}{\left(\alpha_{2}+\lambda_{1}\right)}\right]\right\}, \hat{\bar{b}}_{30}=\frac{\gamma_{2} \theta_{2}}{\left(\alpha_{1}+\gamma_{2}\right)\left(\alpha_{1}+\theta_{1}\right)}\left[\mathrm{p}_{2}+\frac{\mathrm{q}_{2} \lambda_{2}}{\left(\alpha_{1}+\lambda_{2}\right)}\right] \text {, } \\
& \hat{\overline{\mathrm{b}}}_{32}=\frac{1}{\left(\alpha_{1}+\gamma_{2}\right)}\left\{\frac{\gamma_{2} \alpha_{1}}{\left(\alpha_{1}+\theta_{2}\right)}\left[\frac{\mathrm{q}_{2} \theta_{2}}{\left(\alpha_{1}+\lambda_{2}\right)}+1\right]+\alpha_{1}\right\}, \hat{\bar{b}}_{37}=\frac{1}{\left(\alpha_{1}+\gamma_{2}\right)}\left\{1+\frac{\gamma_{2}}{\left(\alpha_{1}+\theta_{2}\right)}\left[1+\frac{\mathrm{q}_{2} \theta_{2}}{\left(\alpha_{1}+\lambda_{2}\right)}\right]\right\} \text {, }
\end{aligned}
$$




$$
\begin{aligned}
& \hat{\overline{\mathrm{b}}}_{21}=\frac{-\gamma_{1} \theta_{1}}{\left(\alpha_{2}+\gamma_{1}\right)\left(\alpha_{2}+\theta_{1}\right)}\left\{\mathrm{p}_{1}\left[\frac{1}{\left(\alpha_{2}+\gamma_{1}\right)}+\frac{1}{\left(\alpha_{2}+\theta_{1}\right)}\right]+\frac{\mathrm{q}_{1} \lambda_{1}}{\left(\alpha_{2}+\lambda_{1}\right)}\left[\frac{1}{\left(\alpha_{2}+\gamma_{1}\right)}+\frac{1}{\left(\alpha_{2}+\theta_{1}\right)}+\frac{1}{\left(\alpha_{2}+\lambda_{1}\right)}\right]\right\} \\
& \hat{\overline{\mathrm{b}}}_{30}=\frac{-\gamma_{2} \theta_{2}}{\left(\alpha_{1}+\gamma_{2}\right)\left(\alpha_{1}+\theta_{2}\right)}\left\{\mathrm{p}_{2}\left[\frac{1}{\left(\alpha_{1}+\gamma_{2}\right)}+\frac{1}{\left(\alpha_{1}+\theta_{2}\right)}\right]+\frac{\mathrm{q}_{2} \lambda_{2}}{\left(\alpha_{1}+\lambda_{2}\right)}\left[\frac{1}{\left(\alpha_{1}+\gamma_{2}\right)}+\frac{1}{\left(\alpha_{1}+\theta_{2}\right)}+\frac{1}{\left(\alpha_{1}+\lambda_{2}\right)}\right]\right\}, \\
& \hat{\overline{\mathrm{b}}}_{32}^{\prime}=\frac{-\gamma_{2} \alpha_{1}}{\left(\alpha_{1}+\gamma_{2}\right)\left(\alpha_{1}+\theta_{2}\right)}\left\{1+\frac{\mathrm{q}_{2} \theta_{2}}{\left(\alpha_{1}+\lambda_{2}\right)}\left[\frac{1}{\left(\alpha_{1}+\theta_{2}\right)}+\frac{1}{\left(\alpha_{1}+\lambda_{2}\right)}+1\right]+\frac{1}{\left(\alpha_{1}+\theta_{2}\right)}+\frac{\mathrm{q}_{2}}{\lambda_{2}}\right\}-\frac{\alpha_{1}}{\left(\alpha_{1}+\gamma_{2}\right)}\left[\frac{1}{\left(\alpha_{1}+\gamma_{2}\right)}+\frac{1}{\theta_{2}}+\frac{\mathrm{q}_{2}}{\lambda_{2}}\right]
\end{aligned}
$$

In this case, $\hat{\mathrm{V}}_{\mathrm{i}}(\mathrm{t})$ are:

$$
\begin{array}{lll}
\hat{V}_{2}(t)=e^{-\left(\alpha_{2}+\gamma_{1}\right) t}, & \hat{V}_{3}(t)=e^{-\left(\alpha_{1}+\gamma_{2}\right) t}, & \hat{V}_{4}(t)=\mathrm{e}^{-\left(\alpha_{2}+\theta_{1}\right) t}, \\
\hat{V}_{5}(t)=e^{-\left(\alpha_{1}+\theta_{2}\right) t}, & \hat{V}_{6}(t)=e^{-\left(\alpha_{2}+\lambda_{1}\right) t}, & \hat{V}_{7}(t)=e^{-\left(\alpha_{1}+\lambda_{2}\right) t}
\end{array}
$$

In long run, the function of time for which the server is busy is given by:

Where:

$$
\hat{\mathrm{B}}_{0}(\infty)=\hat{\mathrm{N}}_{2} / \hat{\mathrm{D}}_{1}
$$

$$
\hat{\mathrm{N}}_{2}=\frac{1}{(\alpha+\gamma)}\left\{\frac{\alpha}{(\theta+\gamma)^{2}}\left[1-\frac{\beta \lambda}{(\beta+\delta)(\lambda+\delta)}\right]+\frac{\beta}{(\lambda+\delta)^{2}(\beta+\delta)}\left[\frac{\alpha \theta(1-\theta)+\alpha \gamma}{\theta(\theta+\gamma)}\right]\right\}
$$

In steady state, number of visits per unit is given by:

Where:

$$
\hat{\mathrm{V}}_{0}(\infty)=\hat{\mathrm{N}}_{3} / \hat{\mathrm{D}}_{2}
$$

$$
\hat{\mathrm{N}}_{3}=\frac{1}{\left(\alpha_{1}+\beta_{1}\right)}\left\{\left[\frac{\beta_{1} \alpha_{2}}{\left(\alpha_{2}+\beta_{2}\right)}+\alpha_{1}\right]\left(1-\hat{\overline{\mathrm{C}}}_{23} \hat{\overline{\mathrm{C}}}_{32}\right)+\frac{\alpha_{1} \alpha_{2} \hat{\overline{\mathrm{C}}}_{21}\left(1-\hat{\overline{\mathrm{C}}}_{32}\right)}{\left(\alpha_{2}+\beta_{2}\right)}\right\}
$$

and:

$$
\begin{aligned}
& \hat{\mathrm{D}}_{2}=\frac{\beta_{1} \beta_{2}\left(1-\hat{\overline{\mathrm{C}}}_{23} \hat{\overline{\mathrm{C}}}_{32}\right)}{\left(\alpha_{1}+\beta_{1}\right)\left(\alpha_{2}+\beta_{2}\right)}\left[\frac{1}{\left(\alpha_{1}+\beta_{1}\right)}+\frac{1}{\left(\alpha_{2}+\beta_{2}\right)}\right]-\left[1-\frac{\beta_{1} \beta_{2}}{\left(\alpha_{1}+\beta_{1}\right)\left(\alpha_{2}+\beta_{2}\right)}\right]\left(\hat{\overline{\mathrm{C}}}_{23}^{\prime} \hat{\overline{\mathrm{C}}}_{30}+\hat{\overline{\mathrm{C}}}_{23} \hat{\overline{\mathrm{C}}}_{30}^{\prime}\right)+\frac{\alpha_{2}}{\left(\alpha_{2}+\beta_{2}\right)} \\
& \left\{\frac{1}{\left(\alpha_{2}+\beta_{2}\right)}\left[\frac{\beta_{1} \hat{\overline{\mathrm{C}}}_{30}}{\left(\alpha_{1}+\beta_{1}\right)}+\hat{\overline{\mathrm{C}}}_{32} \hat{\overline{\mathrm{C}}}_{21}\right]+\frac{\beta_{1}}{\left(\alpha_{1}+\beta_{1}\right)}\left[\frac{\hat{\overline{\mathrm{C}}}_{30}}{\left(\alpha_{1}+\beta_{1}\right)}-\hat{\overline{\mathrm{C}}}_{30}\right]-\hat{\overline{\mathrm{C}}}_{32}^{\prime} \hat{\overline{\mathrm{C}}}_{21}-\hat{\overline{\mathrm{C}}}_{32} \hat{\overline{\mathrm{C}}}_{21}^{\prime}\right\}+\frac{\alpha_{1}}{\left(\alpha_{1}+\beta_{1}\right)} \\
& \left\{\frac{1}{\left(\alpha_{1}+\beta_{1}\right)}\left[\frac{\hat{\overline{\mathrm{b}}}_{21}}{\left(\alpha_{2}+\beta_{2}\right)}\left(\beta_{2}+\alpha_{2} \hat{\overline{\mathrm{b}}}_{30}\right)+\hat{\overline{\mathrm{b}}}_{23} \hat{\overline{\mathrm{b}}}_{30}\right]-\frac{\hat{\overline{\mathrm{b}}}_{21}^{\prime}}{\left(\alpha_{2}+\beta_{2}\right)}\left(\beta_{2}+\alpha_{2} \hat{\overline{\mathrm{b}}}_{30}\right) \frac{\hat{\overline{\mathrm{C}}}_{21}}{\left(\alpha_{2}+\beta_{2}\right)}\left[\frac{\left(\beta_{2}+\alpha_{2} \hat{\overline{\mathrm{C}}}_{30}\right)}{\left(\alpha_{2}+\beta_{2}\right)}-\alpha_{2} \hat{\overline{\mathrm{C}}}_{30}^{\prime}\right]-\hat{\overline{\mathrm{C}}}_{23}^{\prime} \hat{\overline{\mathrm{C}}}_{30}-\hat{\overline{\mathrm{C}}}_{23} \hat{\overline{\mathrm{C}}}_{30}^{\prime}\right\}, \\
& \hat{\overline{\mathrm{C}}}_{21}=\frac{\gamma_{1} \theta_{1}}{\left(\alpha_{2}+\gamma_{1}\right)\left(\alpha_{2}+\theta_{1}\right)}\left[\mathrm{p}_{1}+\frac{\mathrm{q}_{1} \lambda_{1}}{\left(\alpha_{2}+\lambda_{1}\right)}\right], \hat{\overline{\mathrm{C}}}_{23}=\frac{1}{\left(\alpha_{2}+\gamma_{1}\right)}\left\{\frac{\gamma_{1} \alpha_{2}}{\left(\alpha_{2}+\theta_{1}\right)}\left[\frac{\mathrm{q}_{1} \theta_{1}}{\left(\alpha_{2}+\lambda_{1}\right)}+1\right]+\alpha_{2}\right\}, \\
& \hat{\overline{\mathrm{C}}}_{21}^{\prime}=\frac{\theta_{1}}{\left(\alpha_{2}+\theta_{1}\right)}\left\{\frac{\alpha_{1}}{\left(\alpha_{1}+\beta_{1}\right)^{2}}\left[\mathrm{p}_{1}+\frac{\mathrm{q}_{1} \lambda_{1}}{\left(\alpha_{2}+\lambda_{1}\right)}\right]+\frac{\gamma_{1}}{\left(\alpha_{2}+\gamma_{1}\right)}\left[\frac{\mathrm{p}_{1}}{\left(\alpha_{2}+\theta_{1}\right)}+\frac{\mathrm{q}_{1} \lambda_{1}}{\left(\alpha_{2}+\lambda_{1}\right)}\left(\frac{1}{\left(\alpha_{2}+\theta_{1}\right)}+\frac{1}{\left(\alpha_{2}+\lambda_{1}\right)}\right)\right]\right\}, \\
& \hat{\overline{\mathrm{C}}}_{30}=\frac{\gamma_{2} \theta_{2}}{\left(\alpha_{1}+\gamma_{2}\right)\left(\alpha_{1}+\theta_{2}\right)}\left[\mathrm{p}_{2}+\frac{\mathrm{q}_{2} \lambda_{2}}{\left(\alpha_{1}+\lambda_{2}\right)}\right], \hat{\overline{\mathrm{C}}}_{32}=\frac{1}{\left(\alpha_{1}+\gamma_{2}\right)}\left\{\frac{\gamma_{2} \alpha_{1}}{\left(\alpha_{1}+\theta_{2}\right)}\left[\frac{\mathrm{q}_{2} \theta_{2}}{\left(\alpha_{1}+\lambda_{2}\right)}+1\right]+\alpha_{1}\right\},
\end{aligned}
$$




$$
\begin{aligned}
& \hat{\overline{\mathrm{C}}}_{23}^{\prime}=-\frac{\alpha_{2}}{\left(\alpha_{2}+\gamma_{1}\right)}\left\{\frac{\gamma_{1}}{\left(\alpha_{2}+\theta_{1}\right)}\left[\frac{1}{\left(\alpha_{2}+\gamma_{1}\right)}\left(\frac{\mathrm{q}_{1} \theta_{1}}{\left(\alpha_{2}+\lambda_{1}\right)}+1\right)+\frac{\mathrm{q}_{1} \theta_{1}}{\left(\alpha_{2}+\lambda_{1}\right)}\left(\frac{1}{\left(\alpha_{2}+\theta_{1}\right)}+\frac{1}{\left(\alpha_{2}+\lambda_{1}\right)}\right)+\frac{1}{\left(\alpha_{2}+\theta_{1}\right)}+\frac{\mathrm{q}_{1}}{\lambda_{1}}\right]+\frac{1}{\left(\alpha_{2}+\gamma_{1}\right)}+\frac{1}{\theta_{1}}+\frac{\mathrm{q}_{1}}{\lambda_{1}}\right\}, \\
& \hat{\overline{\mathrm{C}}}_{30}^{\prime}=\frac{-\theta_{2}}{\left(\alpha_{1}+\theta_{2}\right)}\left\{\frac{\alpha_{2}}{\left(\alpha_{2}+\beta_{2}\right)^{2}}\left[\mathrm{p}_{2}+\frac{\mathrm{q}_{2} \lambda_{2}}{\left(\alpha_{1}+\lambda_{2}\right)}\right]+\frac{\gamma_{2}}{\left(\alpha_{1}+\gamma_{2}\right)}\left[\frac{\mathrm{p}_{2}}{\left(\alpha_{1}+\theta_{2}\right)}+\frac{\mathrm{q}_{2} \lambda_{2}}{\left(\alpha_{1}+\lambda_{2}\right)}\left(\frac{1}{\left(\alpha_{1}+\theta_{2}\right)}+\frac{1}{\left(\alpha_{1}+\lambda_{2}\right)}\right)\right]\right\} \\
& \hat{\overline{\mathrm{C}}}_{32}^{\prime}=\frac{\alpha_{1}}{\left(\alpha_{1}+\gamma_{2}\right)}\left\{\frac{\gamma_{2}}{\left(\alpha_{1}+\theta_{2}\right)}\left[\frac{1}{\left(\alpha_{1}+\gamma_{2}\right)}\left(\frac{\mathrm{q}_{2} \theta_{2}}{\left(\alpha_{1}+\lambda_{2}\right)}+1\right)+\frac{\mathrm{q}_{2} \theta_{2}}{\left(\alpha_{1}+\lambda_{2}\right)}\left(\frac{1}{\left(\alpha_{1}+\theta_{2}\right)}+\frac{1}{\left(\alpha_{1}+\lambda_{2}\right)}\right)+\frac{1}{\left(\alpha_{1}+\theta_{2}\right)}+\frac{\mathrm{q}_{2}}{\lambda_{2}}\right]+\frac{1}{\left(\alpha_{1}+\gamma_{2}\right)}+\frac{1}{\theta_{2}}+\frac{\mathrm{q}_{2}}{\lambda_{2}}\right\}
\end{aligned}
$$

Table 1: Relation between the failure rate of the operative unit and the

\begin{tabular}{|c|c|c|c|}
\hline \multirow[b]{2}{*}{$\alpha$} & \multicolumn{3}{|l|}{$\mathrm{c}$} \\
\hline & $\gamma=0.2$ & $\gamma=0.5$ & $\gamma=0.8$ \\
\hline 0.05 & 1524.83 & 1150.98 & 944.38 \\
\hline 0.10 & 1495.48 & 1100.01 & 920.75 \\
\hline 0.15 & 1328.60 & 1000.21 & 874.95 \\
\hline 0.20 & 1174.51 & 943.26 & 840.53 \\
\hline 0.25 & 1049.94 & 916.48 & 827.76 \\
\hline 0.30 & 949.44 & 883.24 & 820.59 \\
\hline 0.35 & 886.69 & 845.96 & 807.00 \\
\hline 0.40 & 835.08 & 806.64 & 787.90 \\
\hline 0.45 & 780.45 & 766.81 & 764.55 \\
\hline
\end{tabular}
expected cost per unit time at different value of the rate of repair of the failed unit

Table 2: Relation between the failure rate of the operative unit and

\begin{tabular}{|c|c|c|c|}
\hline \multirow[b]{2}{*}{$\alpha$} & \multicolumn{3}{|l|}{$\mathrm{c}$} \\
\hline & $\lambda=0.2$ & $\lambda=0.5$ & $\lambda=0.8$ \\
\hline 0.05 & 833.95 & 874.47 & 890.28 \\
\hline 0.10 & 813.45 & 870.22 & 887.06 \\
\hline 0.15 & 795.34 & 865.46 & 883.91 \\
\hline 0.20 & 765.26 & 858.92 & 880.37 \\
\hline 0.25 & 724.85 & 852.46 & 875.99 \\
\hline 0.30 & 678.64 & 839.02 & 870.84 \\
\hline 0.35 & 630.55 & 819.29 & 864.67 \\
\hline 0.40 & 583.28 & 794.63 & 861.03 \\
\hline 0.45 & 538.40 & 766.49 & 846.88 \\
\hline
\end{tabular}
the expected cost per unit time at different value of the rate of complete post repair of unsatisfactory failed unit

Numerical example: Let:

$\begin{array}{llll}\beta=0.7, & \theta=0.7, & \mathrm{q}=0.6, & \mathrm{p}=0.4 \\ \lambda=0.5, & \mathrm{~K}_{1}=1000, & \mathrm{~K}_{2}=100, & \mathrm{~K}_{3}=50\end{array}$

Let:

$$
\begin{array}{llll}
\beta=0.6, & \theta=0.7, & \mathrm{q}=0.6, & \mathrm{p}=0.4, \\
\gamma=0.5, & \mathrm{~K}_{1}=1000, & \mathrm{~K}_{2}=100, & \mathrm{~K}_{3}=50
\end{array}
$$

The expected cost per unit time in steady state is:

$$
\hat{\mathrm{C}}=\mathrm{K}_{1} \hat{\mathrm{A}}_{0}-\mathrm{K}_{2} \hat{\mathrm{B}}_{0}-\mathrm{K}_{3} \hat{\mathrm{V}}_{0}
$$

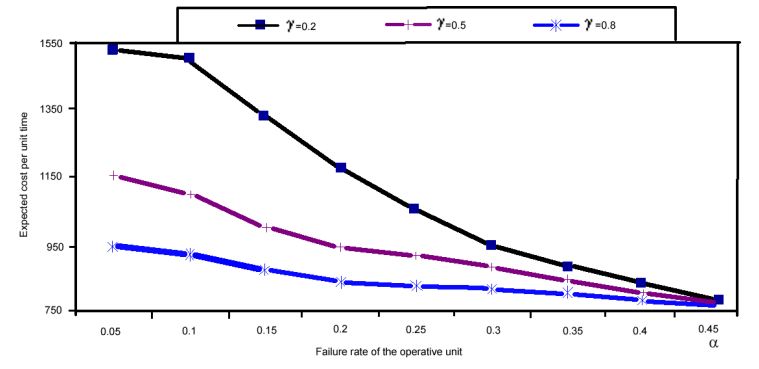

Fig. 2: Relation between the failure rate of the operative unit and the expected cost per unit time

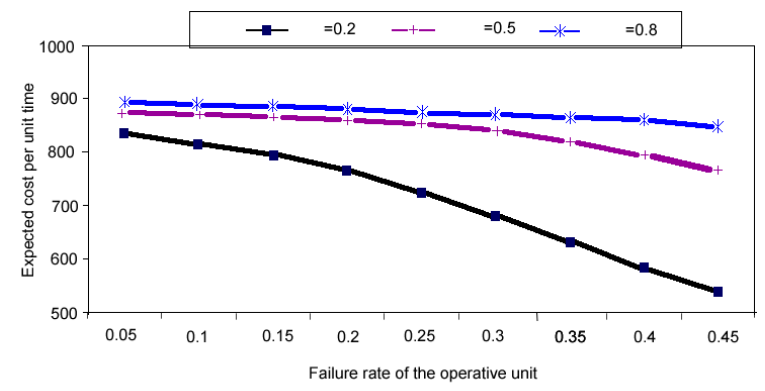

Fig. 3: Relation between the failure rate of the operative unit and the expected cost per unit time

The system was analyzed by semi Markov process technique.

The time-dependent availability, steady-state availability, busy period analysis, expected number of visits by the repairman were obtained numerically and cost analysis was obtained numerically as shown in Table 1 and 2 and graphically and as shown in Fig. 2 and 3.

\section{CONCLUSION}

Table 1 and 2 compute the expected cost per unit time of the system for different values of failure rate $\alpha$. The system is decrease with increase of failure rate $\alpha$ as shown in Fig. 2 and 3. 


\section{REFERENCES}

Goel, L.R., R.K. Agnihotri and R. Gupta, 1992. Profit evaluation of a two unit cold standby system with random change in units. Int. J. Syst. Sci., 24: 367-377. DOI: $10.1080 / 00207729208949212$

Mokaddis, G.S., M.L. Tawfek and S.A.M. Elhssia, 1997. Analysis of a two-dissimilar unit cold standby redundant system subject to inspection and random change in units. Microelect. Reliab. 37: 329-334.

DOI: 10.1016/0026-2714(95)00207-3
Mokaddis, G.S., M.S. El-Sherbiny and E. Al-Sayeh, 2010. Compare between two-unit cold standby and warm standby outdoor electric power systems in charging weather. J. Math. Stat., 6: 17-22. http://www.scipub.org/fulltext/jms2/jms26117-22.pdf 